\title{
Broadband source localization with a single hydrophone
}

\author{
S.M. Jesus ${ }^{1}$, M.B. Porter ${ }^{2}$, Y. Stéphan ${ }^{3}$, E. Coelho ${ }^{4}$ and X. Démoulin ${ }^{3}$ \\ ${ }^{1}$ UCEH - Univ. do Algarve, PT-8000 Faro, Portugal. \\ 2 Dept. of Mathematical Sciences, NJIT, Newark, NJ, USA. \\ ${ }^{3}$ CMO-SHOM, F-29275 Brest Cedex, France. \\ ${ }^{4}$ Instituto Hidrográfico, PT-1296 Lisboa, Portugal.
}

\begin{abstract}
Source localization with vertical arrays in shallow water has been a topic of intense research in the last 20 years. Although horizontal arrays can (and have) been used, vertical arrays are better suited for extracting signal modal structure and thus provide a source-location estimate in range and depth. It is well known that broadband signals have a localization capability superior to that of narrowband signals. One question that remains largely unresolved is whether frequency extent can compensate for the spatial diversity provided by sensor arrays, i.e., at the limit, can a broadband signal be localized with a single sensor ? This communication presents theoretical, simulated, and practical evidence that a multipath-delay maximum-likelihood estimator does provide enough signal to background discrimination for localizing a $500 \mathrm{~Hz}$ bandwidth signal at $5 \mathrm{~km}$ range in a $130 \mathrm{~m}$ depth shallow water channel with a single hydrophone. The real data used in this study was collected during the INTIMATE'96 experiment which was conducted off the west of Portugal in June 1996 during an internal tide tomography experiment. Comparison with localization results provided by direct match between the received and the model-generated arrival patterns will be discussed.
\end{abstract}

Keywords - Source localization, subspace methods, shallow water, broadband.

\section{INTRODUCTION}

Classical matched-field processing (MFP) methods mostly use vertical or horizontal hydrophone arrays with significant apertures in order to obtain sufficient source location spatial discrimination. Single sensor localization was first presented by Frazer [1] based on a technique suggested by Clay [2]. In both of these studies, results were obtained using simulated data by reverse correlation of the received time-series with the model predicted received signal. The lack of spatial aperture was compensated for by full time- domain processing. Time domain matching is highly dependent on the ability of the numerical models to predict actual received signal amplitude and, more importantly, the signal phase. In order to avoid the dependence on exact phase prediction, incoherent MFP has been suggested [3] and successfully applied on real data (for example in [4],[5]). Time domain source localization was actually achieved by Brienzo et al. [6] using data received on a vertical array in a deep water area on the Monterey fan. In this case a generalized conventional beamformer was used for recombining the received data in time domain (matched-filter) and then between sensors in space domain (beamformer). Evidence of single sensor time-domain source localization and tracking has been demonstrated recently by Porter et all. [7],[8]. This paper presents two types of methods: the first type computes the classical maximum- likelihood estimate of the channel multipath arrival times and searches for the source location for which the time delay set best matches the estimated arrival times. The solution is equivalent to a maximum-likelihood estimate of the source location constrained to the given acoustic model. The second type of methods makes the further assumption that there are features (like clusters of acoustic arrivals) that are decorrelated, allowing for signal-noise subspace splitting. In that case estimating source location is equivalent to measuring the distance between the estimated signal subspace and the subspace spanned by the delayed source signal paths given by the acoustic model. This paper compares the results obtained by those methods and discusses differences with those obtained in [7],[8].

\section{LINEAR DATA MODEL}

According to the linear data model, the received acoustic signal due to a source at location $\theta_{s}=\left(r_{s}, z_{s}\right)$ is given by

$$
y_{n}\left(t, \theta_{s}\right)=p_{n}\left(t, \theta_{s}\right) * s_{0}(t)+\epsilon_{n}(t),
$$

where $p$ is the channel impulse response, $s_{0}$ is the waveform transmitted by the source and $\epsilon$ is the noise sequence, assumed spatially and temporally white, zeromean and uncorrelated with the signal. Under the assumption that the medium between the source and the receiver behaves as a multiple time delay-attenuation channel, its impulse response can be written

$$
p_{n}\left(t, \theta_{s}\right)=\sum_{m=1}^{M} a_{n, m}\left(\theta_{s}\right) \delta\left[t-\tau_{m}\left(\theta_{s}\right)\right],
$$


where the $\left\{a_{n, m}\left(\theta_{s}\right)\right\}$ and $\left\{\tau_{m}\left(\theta_{s}\right)\right\}$ are respectively the signal attenuations and time delays along the $M$ acoustic paths at time snapshots $n=1, \ldots, N$. In (2), the time delay variability within each data set of $N$ snapshots has been neglected. In a more concise matrix notation (1) can be written as

$$
\mathbf{y}_{n}\left(\theta_{s}\right)=\mathbf{S}\left[\boldsymbol{\tau}\left(\theta_{s}\right)\right] \mathbf{a}_{n}\left(\theta_{s}\right)+\boldsymbol{\epsilon}_{n},
$$

with

$$
\begin{gathered}
\boldsymbol{\tau}\left(\theta_{s}\right)=\left[\tau_{1}\left(\theta_{s}\right), \ldots, \tau_{M}\left(\theta_{s}\right)\right]^{t}, \\
\mathbf{S}\left[\boldsymbol{\tau}\left(\theta_{s}\right)\right]=\left[\mathbf{s}_{0}\left(\tau_{1}\right), \ldots, \mathbf{s}_{0}\left(t-\tau_{M}\right)\right], \\
\mathbf{a}_{n}\left(\theta_{s}\right)=\left[a_{n, 1}\left(\theta_{s}\right), \ldots, a_{n, M}\left(\theta_{s}\right)\right]^{t}, \\
\mathbf{s}_{0}(\tau)=\left[s_{0}(-\tau), \ldots, s_{0}(T \Delta t-\tau)\right]^{t},
\end{gathered}
$$

where $T$ is the number of time samples in the emitting waveform and $\Delta t$ is the sampling interval. Equation (3) forms a linear model on the amplitude vector $\mathbf{a}$, where further assumptions on the relative dimensions and rank of matrix $\mathbf{S}$ and noise distributions allow for different solutions for the estimation of $\boldsymbol{\tau}$.

\section{TIME DELAY AND AMPLITUdE ESTIMATORS}

Estimating $\boldsymbol{\tau}$ in (3) is a sort of classical two-step problem that may be easily addressed using least squares (LS), or under the Gaussian white noise assumption, as a generalized maximum likelihood (ML) problem. In either case, the first step consists of the estimation of a that is: determine $\hat{\mathbf{a}}$ that minimizes

$$
e(\boldsymbol{\tau}, \mathbf{a})=\|\mathbf{y}-\mathbf{S}(\boldsymbol{\tau}) \mathbf{a}\|^{2},
$$

whose solution is well-known to be

$$
\hat{\mathbf{a}}=\left(\mathbf{S}^{H} \mathbf{S}\right)^{-1} \mathbf{S}^{H} \mathbf{y},
$$

where ${ }^{H}$ indicates complex conjugate transpose. The second step proceeds by plugging (6) into (5) and gives a new function to be minimized,

$$
e(\boldsymbol{\tau})=\left\|\mathbf{y}^{H} \mathbf{S}(\boldsymbol{\tau})\right\|^{2},
$$

which is now only a function of delay vector $\tau$. Passing from $(5)$ to $(7)$, requires the additional assumption that the matrix $\mathbf{S}$ is orthogonal, i.e., that $\mathbf{S}^{H} \mathbf{S}=\mathbf{I}$. In terms of propagation, that assumption is equivalent to assuming that signals travelling along different paths suffer uncorrelated perturbations. Whether this occurs in practice depends on a variety of factors. The description above assumes that only a single measurement $\mathbf{y}$ is available. If instead there are $N$ randomly distributed vectors $\mathbf{Y}=\left[\mathbf{y}_{1}, \ldots, \mathbf{y}_{N}\right]$, a formulation similar to that followed above leads to

$$
e(\boldsymbol{\tau})=\frac{1}{N} \sum_{n=1}^{N}\left\|\mathbf{y}_{n}^{H} \mathbf{S}(\boldsymbol{\tau})\right\|^{2} .
$$

In this case, one can estimate the $M$ time delays from the $M$ highest peaks of function (8), i.e.,

$$
\left\{\hat{\tau}_{m}^{\mathrm{LS}} ; m=1, \ldots, M\right\}=\arg \left\{\max _{\tau} \sum_{n=1}^{N}\left\|\mathbf{y}_{n}^{H} \mathbf{s}_{0}(\tau)\right\|^{2}\right\} \text {. }
$$

Once model (3) has been adopted, an additional assumption on the mutual decorrelation of the multipath amplitudes (assumed as random and zero mean), allows one to extend the LS/ML method above, to subspace (SS) separation based methods. In fact, the linear model (3) allows one to characterize the signal part as covering a $K(<M)$-dimensional subspace where $K$ is the number of uncorrelated paths (or groups of paths) in the received signal-this is the signal subspace. It can be shown[9] that the $M$ eigenvectors $\left\{\mathbf{u}_{1}, \ldots, \mathbf{u}_{M}\right\}$ associated with the $M$ largest singular values $\left\{\sigma_{1}, \ldots, \sigma_{M}\right\}$ of data matrix $\mathbf{Y}$ provide the optimal estimate of the signal subspace. Indeed the vectors $\mathbf{u}_{m} ; m=1, \ldots, M$ span the same ( $\mathrm{sig}$ nal) subspace as the $M$ signal replicas $\mathbf{s}\left(\tau_{1}\right), \ldots, \mathbf{s}\left(\tau_{M}\right)$. Therefore, considered as a function of search delay $\tau$, the projection of the signal replicas onto the subspace spanned by the first $M$ eigenvectors will be a maximum for $\tau=\tau_{m} ; m=1, \ldots, M$. Thus we seek the maxima of the functional

$$
e(\tau)=\left\|\mathbf{U}_{M}^{H} \mathbf{s}_{0}(\tau)\right\|^{2},
$$

where $\mathbf{U}_{M}=\left[\mathbf{u}_{1}, \ldots, \mathbf{u}_{M}\right]$. Using (10), the associated signal subspace (SS) based time delay $\tau_{m}$ estimator can be written

$$
\left\{\hat{\tau}_{m}^{\mathrm{SS}} ; m=1, \ldots, M\right\}=\arg \left\{\max _{\tau}\left\|\mathbf{U}_{M}^{H} \mathbf{s}_{0}(\tau)\right\|^{2}\right\} .
$$

Similarly, knowing that $\mathbf{U}_{M}$ and its complement $\mathbf{U}_{T-M}=\mathbf{U}_{M}^{\perp}$ split the whole space $\Re^{T}$ into two orthogonal subspaces, the projection of the signal replicas onto the $\mathbf{U}_{M}$ subspace complement will tend to zero for the same true values of $\tau$. Therefore, the noise subspace based time delay $\tau_{m}$ estimator is given by

$\left\{\hat{\tau}_{m}^{\mathrm{SS}}{ }^{\perp} ; m=1, \ldots, M\right\}=\arg \left\{\max _{\tau}\left[\left\|\mathbf{U}_{T-M}^{H} \mathbf{s}_{0}(\tau)\right\|^{2}\right]^{-1}\right\}$,

where the matrix $\mathbf{U}_{T-M}=\left[\mathbf{u}_{M+1}, \ldots, \mathbf{u}_{T}\right]$ is formed from the data eigenvectors associated with the $M+1$ to $T$ lowest eigenvalues. These eigenvectors span the subspace containing the non-signal components so the estimator is generally called the noise subspace estimator.

\section{SOURCE LOCALIZATION}

The source localization problem can be readily deduced from the last section both for the LS/ML and the subspace splitting methods. Until now only the received signal was used for analysis but source localization requires 
data inversion for source properties. That means, in particular, that the medium where the signal is propagating has to be taken into account using a specific propagation model to solve the forward problem. The propagation model determines a set of time delays at the receiver for the given environment and for each hypothetical source location. Let us define $\boldsymbol{\tau}(\theta)$ as the model-calculated time delay vector for source location $\theta$, conditioned on a given environmental scenario. For all possible values of $\theta$ in a set $\Theta$, the vector $\boldsymbol{\tau}(\theta)$ will cover a continuum on an $M$ dimensional space as does the source replica vector. A constrained LS/ML based estimate $\hat{\theta}_{\text {MLS }}$ of source location $\theta_{s}$ will be, according to (8)-(9), given by the value of $\theta$ that satisfies

$$
\max _{\boldsymbol{\tau}(\theta)} \frac{1}{N} \sum_{n=1}^{N}\left\|\mathbf{S}[\boldsymbol{\tau}(\theta)]^{H} \mathbf{y}_{n}\right\|^{2}, \quad \theta \in \Theta .
$$

The resulting source location estimator can therefore be written as

$$
\hat{\theta}_{\mathrm{LS}}=\arg \left\{\max _{\boldsymbol{\tau}(\theta)} \sum_{n=1}^{N} \sum_{m=1}^{M}\left\|\mathbf{s}_{0}\left[\tau_{m}(\theta)\right]^{H} \mathbf{y}_{n}\right\|^{2}\right\}, \quad \theta \in \Theta .
$$

Similarly starting from (11) for the SS approach, the source location estimate corresponds to the maximum of the sum over paths of the projections of the replica signal for each time delay set onto the estimated signal subspace,

$$
\hat{\theta}_{\mathrm{SS}}=\arg \left\{\max _{\boldsymbol{\tau}(\theta)} \sum_{m=1}^{M}\left\|\mathbf{U}_{M}^{H} \mathbf{s}_{0}\left[\tau_{m}(\theta)\right]\right\|^{2}\right\}, \quad \theta \in \Theta .
$$

Finally, for the $\mathrm{SS}^{\perp}$ approach, the ambiguity function is searched for the minimum of the sum over paths of the projections onto the noise subspace estimate,

$$
\hat{\theta}_{\mathrm{SS}^{\perp}}=\arg \left\{\min _{\boldsymbol{\tau}(\theta)} \sum_{m=1}^{M}\left\|\mathbf{U}_{T-M}^{H} \mathbf{s}_{0}\left[\tau_{m}(\theta)\right]\right\|^{2}\right\}, \quad \theta \in \Theta .
$$

\section{REAL DATA RESUlTS}

The INTIMATE'96 sea trial was primarily designed as an acoustic tomography experiment to observe internal tides[12]. That experiment took place on the continental shelf near the town of Nazaré, off the west coast of Portugal, during June 1996. The experimental scenario during phase 1 of the experiment is schematically shown in Fig.1. The scenario involves an approximately rangeindependent $135 \mathrm{~m}$ depth waveguide excited at one end by a stationnary sound source. The source is at $92 \mathrm{~m}$ depth and emits a $2 \mathrm{~s}$ long 300-800 Hz LFM sweep which is received at the other end on a single hydrophone located at $115 \mathrm{~m}$ depth and at $5.5 \mathrm{~km}$ range. The environment is characterized by a downward refracting profile

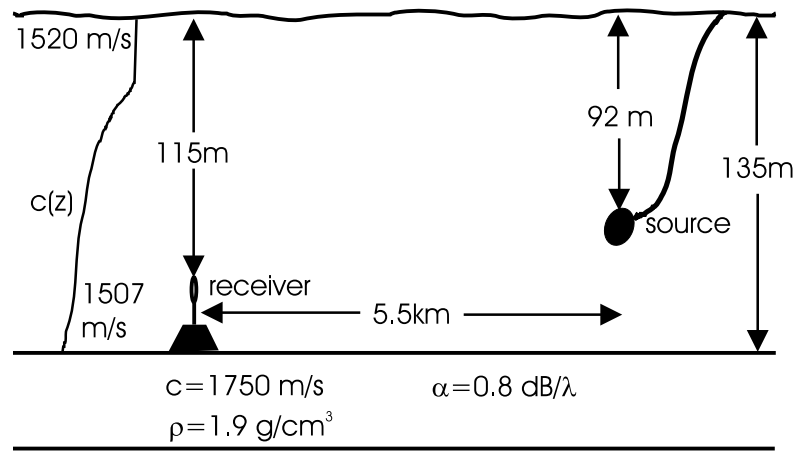

Fig. 1. Experimental scenario for phase 1 of INTIMATE'96.

Over a sandy hott.om with $1750 \mathrm{~m} / \mathrm{s}$ sonind sneed a den-

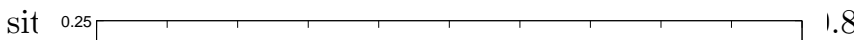
$\mathrm{dF}$

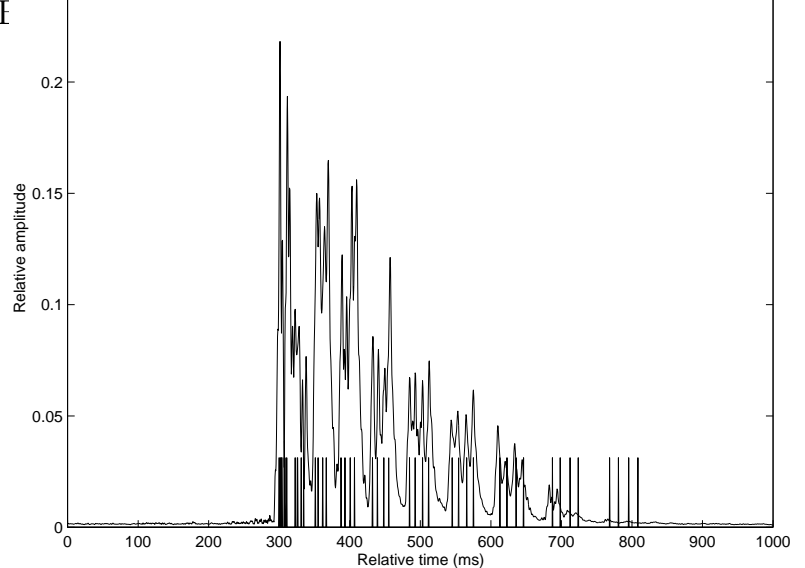

Fig. 2. Arrival pattern using the LS estimator for a sound source at $5.5 \mathrm{~km}$ range and $92 \mathrm{~m}$ depth received on a sensor at $115 \mathrm{~m}$ depth. Vertical lines on time axis represent Bellhop predicted arrival times.

The received pulses are processed using the LS estimator (9) with $N=35$, to obtain the arrival pattern as shown on Fig.2. It can be observed that the emitted pulse is subject to a strong multipath effect extending for more than 0.5 seconds. Figure 2 also shows, the corresponding Bellhop[10] predicted arrival times that are represented by the vertical lines on the time axis below the arrival pattern. The agreement between the peaks of the arrival pattern and the predicted ray arrival-times is nearly perfect for this case.

The first problem encountered when processing the real data using the subspace based methods was the estimation of the number of existing paths, $M$, in equations (11)-(12) and (15)-(16). In principle, $M$ can be predicted by the acoustic model for each source range and should be equal to the rank of matrix $\mathbf{S}$. However, in practice, it was found that the matrix $\mathbf{S}$ was largely rank deficient, and the number of estimated uncorrelated paths (or path groups) was much smaller than the number of predicted 


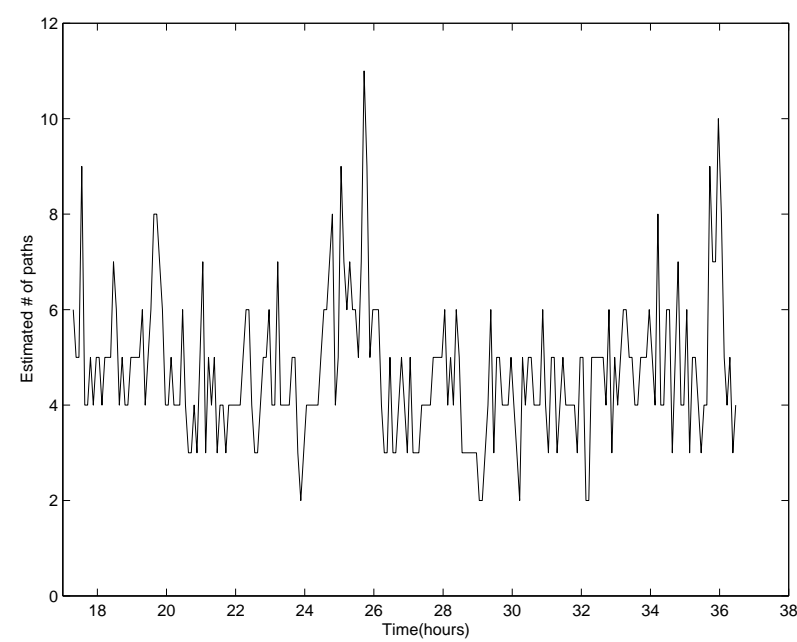

Fig. 3. Estimated number of uncorrelated paths: with Akaike's (AIC) criterion. The start time is 17:20 June 14, 1996.

paths $M$. Figure 3 shows the number of estimated paths for a 20 hour long run using the classical Akaike Information Criterion (AIC) [11]. It can be seen that the estimated number of paths varies from 4 to 5 for AIC while the exnected (a.t least from the rav-model nredic-
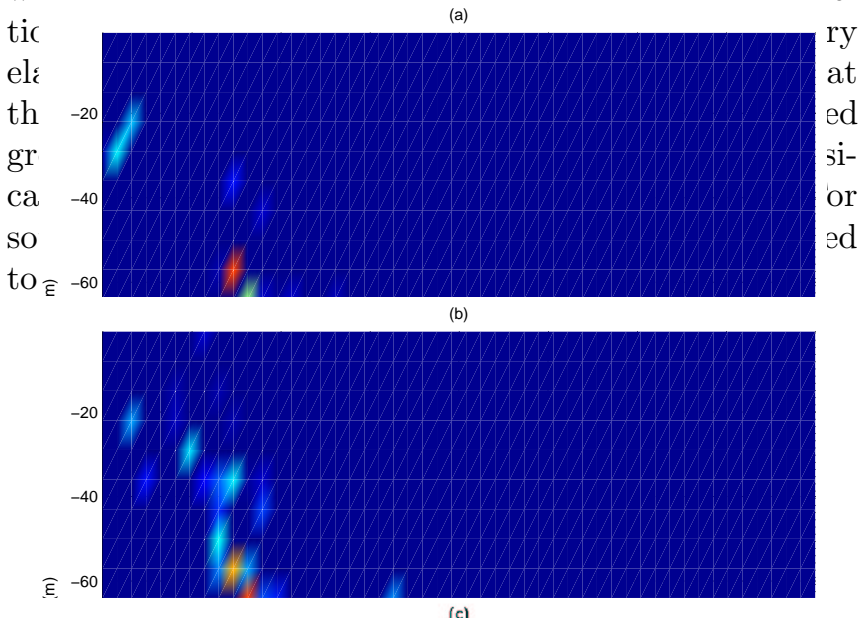

(c)

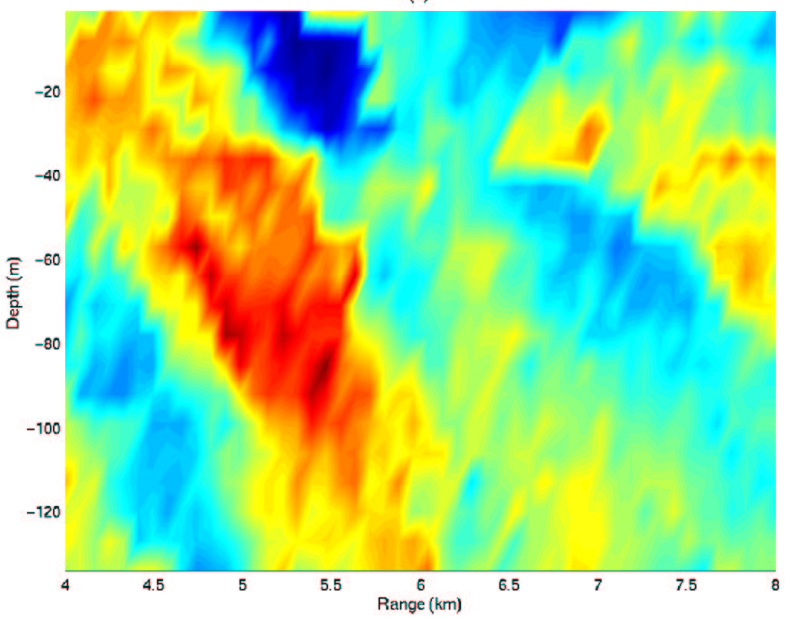

Fig. 4. Range-depth ambiguity surfaces with the following estimators: least squares, LS - eq. (14), (a), signal subspace, SS - eq. (15), (b), and noise subspace, $\mathrm{SS}^{\perp}$, eq.(16), (c).

\section{TABLE I}

INTIMATE'96 experiment, phase 1: estimated mean source range and depth using estimators least squares (LS), eq.(14), signal subspace (SS), eq.(15) and noise sub- space, $\mathrm{SS}^{\perp}$, eq.(16).

\begin{tabular}{lcccc}
\hline & \multicolumn{2}{c}{$\begin{array}{c}\text { range } \\
(\mathrm{km})\end{array}$} & \multicolumn{2}{c}{$\begin{array}{c}\text { depth } \\
(\mathrm{m})\end{array}$} \\
\hline & mean & std & mean & std \\
\cline { 2 - 5 } $\mathrm{LS} / \mathrm{ML}$ & 5.2 & 0.55 & 81 & 35 \\
$\mathrm{SS}$ & 5.2 & 0.54 & 91 & 38 \\
$\mathrm{SS}^{\perp}$ & 4.99 & 0.42 & 63 & 30 \\
\hline
\end{tabular}

As an example the results obtained for simultaneous range-depth estimation are shown on Fig.4. For this particular run all three estimators provided approximately the correct source location at $92 \mathrm{~m}$ depth and $5.4 \mathrm{~km}$ range. This was not always the case during the whole data set, which lasted for over 20 hours. Table 1 shows the mean localization results and the associated standard deviations for all three estimators. Apart from a small bias, source range was relatively well retrieved while source depth has a large standard deviation. However, if we separate the range-depth estimation problem and consider the estimation of depth at known range, we get accurate estimates of depth as well[13].

\section{Discussion AND CONCLUSIONS}

The same data set has served for the results presented by Porter et al. [7],[8] and those presented here. The method proposed by Porter is based on the correlation of the logarithm of the received arrival pattern (9) and the logarithm of the predicted arrival pattern. The resulting estimator accentuates the basic arrival pattern (in terms of arrival times) rather than the arrival amplitude. However, as the processor is based on a correlation of the complete time-series it is sensitive to both the peaks and valleys of the data. In the present study, greater emphasis is placed on the arrival-times of the individual paths. In fact, the match function given by (14) is made only for the predicted arrival times. In other words, only the peaks of the arrival pattern (assuming the correct prediction of time delays) are used. Obviously, the result will be optimal if the peak locations are correctly predicted and resolved, and this is why subspace methods have been introduced for time-delay resolution enhancement. This paper has also shown that time delays can be derived from the intersection of the signal subspace estimate and the subspace spanned by the replica signals. These method, used in conjunction with a suitable acoustic model conditioned on the environmental information, lead to estimators of source location. These source location estimators have been applied to a sound source emitting a 300-800 Hz, 2 seconds long FM sweep, in a shallow water area off the coast of Portugal. The approximate source range, and depth, were successfully estimated during the runs where the source was held approximately fixed. To some extent, these results confirm those obtained by Porter and both studies show the feasi- 
bility of single sensor source localization. Those obtained with the subspace methods shed a new light into multipath correlation and could be even more usefull in cases of unresolvable multipath arrivals. This is particularly important in shallow water scenarios and/or with narrower frequency band waveforms.

\section{ACKNOWLEDMEnTS}

This work was partially supported by PRAXIS XXI program under project 2./2.1/MAR/1695/95. The authors would like to thanks SACLANTCEN for lending the acoustic receiving system and the dedicated collaboration of P. Felisberto in the real data acquisition and processing during INTIMATE'96.

\section{REFERENCES}

[1] L.Neil Frazer and P.I. Pecholcs, "Single-hydrophone localization", J. Acoust. Soc. Am., vol. 88(2), pp.995-1002, 1990.

[2] C.S. Clay, "Optimum time domain signal transmission and source location in a waveguide", J. Acoust. Soc. Am., vol. 81, pp.660-664, 1987.

[3] A.B. Baggeroer, W.A. Kuperman and H. Schmidt, "Matchedfield processing: source localization in correlated noise as an optimum parameter estimation problem", J. Acoust. Soc. Am., vol. 83, 571-587, 1988.

[4] S.M. Jesus, "Broadband matched-field processing of transient signals in shallow water", J. of Acoust. Soc. Am., vol. 93(4), Pt. 1, pp.1841-1850, 1993.

[5] S.P. Czenszak and J.L. Krolik, "Robust wideband matchedfield processing with a short vertical array", J. Acoust. Soc. Am., vol. 101(2), pp.749-759, 1997.

[6] R.K. Brienzo and W. Hodgkiss, "Broadband matched-field processing", J. Acoust. Soc. Am., vol. 94(5), pp. 2821-2831, 1993.

[7] M.B. Porter, S. Jesus, Y. Stéphan, X. Démoulin and E. Coelho, "Exploiting reliable features of the ocean channel response", Proc. of SWAC'97, Beijing, April 1997.

[8] M.B. Porter, Y. Stéphan, X. Démoulin, S. Jesus and E. Coelho, "Shallow-water tracking in the sea of Nazaré", Proc. Underwater Technologies'98, IEEE Oceanic Engineering Society, Tokyo, Japan, 1998.

[9] O.R. Schmidt A Signal Subspace Approach to Multiple Emitter Location and Spectral Estimation, Ph.D. Thesis, Stanford University, 1982.

[10] M.B. Porter and Y.C. Liu, "Finite-Element Ray Tracing", Proc. Int. Conf. on Theoretical Comp. Acoust., Vol.2, pp. 947956, ed. D. Lee \& M.H. Schultz, World Scientific, 1993.

[11] M. Wax and T. Kailath, "Detection of signals by information theoretic criteria", IEEE Trans. on Acoust. Speech and Signal Processing, Vol. ASSP-33, no.2, 1985.

[12] X. Démoulin, Y.Stéphan, S. Jesus, E.Coelho and M.B. Porter, "INTIMATE96: a shallow water tomography experiment devoted to the study of internal tides", Proc. of SWAC'97, Beijing, April 1997.

[13] S.M. Jesus, M.B. Porter, Y. Stéphan, X. Démoulin, O. Rodriguez and E. Coelho, "Single hydrophone source localization", submitted to IEEE Journal of Oceanic Engineering, June 1998. 